\title{
Estudo prospectivo observacional sobre a incidência de Injúria Renal Aguda em Unidade de Terapia Intensiva de um Hospital Universitário
}

\author{
Observational prospective study on the incidence of acute renal \\ failure in the intensive care unit of a university-affiliated hospital
}

\section{Autores}

Nara Yamane dos Santos

Caroline de Pietro Franco Zorzenon

Márcio Fernando de Araújo

André Luís Balbi

Daniela Ponce

Departamento de Clínica Médica da Faculdade de Medicina de Botucatu - UNESP
Data de submissão: 08/06/2009 Data de aprovação: 17/08/2009

Correspondência para: Daniela Ponce Ângelo Simonetti, 366 Botucatu - SP CEP: $18608-392$ Tel: (14) 3882-6873

Declaramos a inexistência de conflitos de interesse.

\section{Resumo}

Objetivo: Analisar comparativamente características clínicas e evolução de pacientes com e sem IRA adquirida em UTI geral de um hospital universitário terciário. Método: Estudo prospectivo observacional com 263 pacientes acompanhados diariamente durante a internação em UTI Geral do Hospital das Clínicas da Faculdade de Medicina de Botucatu no período de julho de 2007 a abril de 2008. Resultados: A incidência de IRA foi de 31,2\%. Os grupos foram semelhantes quanto ao sexo e diferiram quanto à etiologia da admissão em UTI (sepse: $31,7 \%$ x 13,1\%, $\mathrm{p}<0,0001$, pós-operatório: $11 \% \times 43 \%$, $\mathrm{p}<0,0001)$, idade $(59,6 \pm 18,1 \times 50,2 \pm$ 18,6 anos, $\mathrm{p}<0,0001)$, APACHE II: $(21$ $\pm 11,1 \times 11 \pm 4,8, \mathrm{p}=0,002)$, oligúria $(67,7 \% \times 4,5 \%, p<0,0001)$, presença de ventilação mecânica $(81,7 \times 57,7 \%, \mathrm{p}=$ $0,0014)$, uso de drogas vasoativas $(62,2$ $\mathrm{x} 32,6 \%, \mathrm{p}<0,0001)$ e enfermaria de procedência (PS: $22 \times 14,5 \%, \mathrm{p}=0,02 \mathrm{e}$ centro cirúrgico: $42,7 \times 62,6 \%, p=0,03$ ). Quanto às comorbidades, os grupos foram diferentes quanto à presença de HAS e IRC $(42,6 \times 35,9 \%, p=0,005$ e 15,8 x $2,1 \%, p=0,04$, respectivamente) e semelhantes quanto à presença de diabetes e ICC $(19,5 \times 11 \%$, ns e $6 \times 1,1 \%$, ns, respectivamente). A mortalidade foi superior nos pacientes que contraíram IRA $(62,1$ x 16,5\%, p < 0,0001). Conclusão: A incidência de IRA é elevada em UTI e presente em pacientes com parâmetros clínicos e índices prognósticos de maior gravidade, o que justifica a maior mortalidade observada neles.

Palavras-chave: injúria renal aguda, incidência, unidade de terapia intensiva, mortalidade, diálise.

[J Bras Nefrol 2009;31(3):206-211]@Elsevier Editora Ltda.

\section{Abstract}

Objective: To compare the clinical characteristics and outcomes of patients with and without acute renal failure (ARF) admitted to the general intensive care unit (ICU) of a university-affiliated hospital. Method: Prospective observational study comprising 263 patients followed up daily during their stay at the general ICU of the Hospital das Clínicas of the Medical School of Botucatu, from July 2007 to April 2008. Results: The incidence of ARF was $31.2 \%$. The groups were similar regarding gender and differed regarding the following: cause of ICU admission (sepsis: $31.7 \%$ x $13.1 \%$, $\mathrm{p}<0.0001$, post-operative period: $11 \% \mathrm{x}$ $43 \% ; \mathrm{p}<0.0001)$; age $(59.6 \pm 18.1 \times 50.2$ \pm 18.6 years; $\mathrm{p}<0.0001)$; APACHE II: $(21 \pm 11.1 \times 11 \pm 4.8 ; \mathrm{p}=0.002)$; oliguria $(67.7 \% \times 4.5 \%$; $<<0.0001)$; presence of mechanical ventilation $(81.7 \%$ x $57.7 \%$; $=0.0014)$; use of vasoactive drugs $(62.2 \%$ x $32.6 \%$; p < 0.0001); and site of origin inside hospital (emergency room: $22 \% \mathrm{x}$ $14.5 \% ; \mathrm{p}=0.02$; operating room: $42.7 \% \mathrm{x}$ $62.6 \% ; p=0.03)$. As for comorbidities, hypertension and chronic renal disease were more frequent among patients with ARF than among those without ARF $(42.6 \%$ x $35.9 \%$; $=0.005$ and $15.8 \%$ x $2.1 \%$; $\mathrm{p}=0.04$, respectively), but the groups did not differ regarding diabetes and congestive heart failure $(19.5 \% \times 11 \%$, and $6 \% \times 1.1 \%$, respectively). Mortality was higher in ARF patients $(62.1 \% \times 16.5 \%$; $p<0.0001)$. Conclusion: The incidence of ARF is high in the ICU and among patients with worse clinical parameters and prognosis, justifying the greater mortality observed in such patients.

Keywords: acute renal failure, incidence, intensive care unit, mortality, dialysis. 


\section{INTRODUÇÃO}

A Injúria Renal Aguda (IRA) se caracteriza por uma rápida queda do ritmo de filtração glomerular, podendo ser acompanhada de retenção de produtos nitrogenados e distúrbios hidroeletrolíticos. ${ }^{1-3}$ Trata-se de uma patologia complexa, de etiologias múltiplas e variáveis e sem consenso em sua definição. ${ }^{4}$

Em trabalho publicado por Ricci et al.,$^{5}$ há mais de duzentas definições distintas utilizadas pelos nefrologistas. Por outro lado, a dificuldade em se desenvolver uma definição de consenso resulta da ausência de marcadores ideais e capazes de detectar injúria renal em fases muito precoces., ${ }^{4,5}, \mathrm{Em} 2007$, Mehta et al. ${ }^{7}$ publicaram estudo que teve como objetivo principal a tentativa de uniformizar o diagnóstico e a classificação da IRA, propondo, como critérios diagnósticos da IRA, alterações agudas dos níveis séricos da creatinina (aumento absoluto de creatinina superior a $0,3 \mathrm{mg} / \mathrm{dL}$ ou relativo de $50 \%$ em relação ao valor basal) ou do débito urinário (diminuição inferior a $0,5 \mathrm{~mL} / \mathrm{kg} / \mathrm{min}$ por mais de seis horas - oligúria). Embora haja consenso de que a dosagem da creatinina sérica não é um teste ideal para o diagnóstico, não foi possível, até o momento, a inclusão de outros testes mais precisos na prática clínica. ${ }^{8-11}$

A IRA é uma das mais importantes complicações observadas em pacientes que estão hospitalizados. Sua incidência varia de acordo com as condições clínicas dos pacientes, sendo maior em Unidades de Terapia Intensiva (UTI - 20 a 40\%) e menor em unidades em que o cuidado é intermediário ( 1 a $7 \%$ ). Em recente estudo multicêntrico, foi verificada a incidência de 5,7\% de IRA com necessidade de diálise em pacientes internados em UTI. ${ }^{12}$ Além de representar complicação comum em pacientes críticos, é um fator de risco independente de morte. ${ }^{1,3,8,12}$

Nas últimas décadas, tem-se presenciado incansável busca pela redução da mortalidade de pacientes com IRA. A despeito de todos os esforços em busca da terapêutica ideal, a mortalidade geral dos pacientes com IRA não melhorou significativamente nos últimos anos. Vários estudos mostram que a mortalidade geral na IRA permanece próxima a $50 \%$, chegando a $80 \%$ nos pacientes admitidos em UTI. ${ }^{12-14}$ Santos et al. ${ }^{15}$ em estudo nacional de publicação recente, mostrou que a mortalidade dos pacientes com IRA do tipo necrose tubular aguda admitidos em UTI geral de um hospital universitário foi de $62 \%$.

Muitos fatores contribuem para a manutenção da mortalidade elevada na IRA, com destaque pa- ra a falta de identificação de fatores de risco para o desenvolvimento dessa patologia, assim como o diagnóstico tardio e o desconhecimento de fatores associados à mortalidade. ${ }^{15}$

De modo geral, estudos da literatura revelam que a presença de condições que determinam hipoperfusão e isquemia renal está diretamente relacionada com o desenvolvimento de IRA, e os pacientes que apresentam redução da reserva funcional renal são mais suscetíveis a desenvolver IRA, mesmo com lesões renais de pequena monta. ${ }^{16,17}$ Consideram-se fatores de risco para IRA idade avançada, nível prévio de creatinina, presença de diabetes mellitus, de hipertensão arterial e de insuficiência cardíaca congestiva, além do uso crônico de anti-inflamatórios não hormonais. ${ }^{16,17}$

Outra possível explicação para a manutenção da alta mortalidade na IRA é a mudança no perfil epidemiológico desses pacientes, que se tornaram mais graves com o predomínio de insuficiência de múltiplos órgãos e sistemas, estado, em geral, associado à elevada mortalidade. Nessa condição clínica, raramente a IRA apresenta-se como manifestação isolada, mas sim como uma complicação de largo espectro de doenças. ${ }^{18-20}$ Dessa maneira, o desenvolvimento de complicações durante a internação em UTI, como infecções, sepse, hemorragias, cirurgias e necessidade de diálise, pode fazer com que o nível de severidade do paciente e da IRA seja maior. Registre-se que esses fatores estão associados à maior mortalidade. ${ }^{18-20} \mathrm{Mac}-$ cariello et al., ${ }^{21}$ em estudo recém-publicado, concluíram que idade avançada, insuficiência de múltiplos órgãos e sistemas, presença de comorbidades e necessidade de diálise no primeiro dia após a admissão em UTI são fatores de mau prognóstico.

No Hospital das Clínicas da Faculdade de Medicina de Botucatu (HC-FMB), a incidência de IRA é de cerca de $1,9 \%$ das internações. ${ }^{22}$ Entretanto, nas UTIs do HC, estima-se que essa incidência apresente aumento para $40 \%$, com mortalidade dos pacientes em torno de $70 \%$. É consenso geral da equipe que presta o atendimento nefrológico que provavelmente vários diagnósticos de IRA não são realizados ou ocorrem de modo tardio. O grande impacto da IRA em pacientes sob cuidado de UTI leva à necessidade de determinar seu comportamento nessa população, fator que impulsiona a elaboração deste trabalho, que consiste em um estudo exploratório que visa à identificação de sua incidência, assim como à análise das características clínicas desses pacientes, para que medidas preventivas e de diagnóstico precoce possam ser realizadas. ${ }^{23,24}$ 


\section{Objetivos}

\section{Os objetivos deste trabalho são:}

- avaliar a incidência de IRA em pacientes admitidos nos leitos da UTI central do HC-FMB durante um período definido, com base nos critérios diagnósticos adotados pelo Grupo de Atendimento da IRA da Disciplina de Nefrologia da FMB-UNESP;

- analisar comparativamente características clínicas e a evolução de pacientes com e sem IRA adquirida na UTI central;

- verificar a mortalidade de pacientes que desenvolvem IRA quando comparados com aqueles que não desenvolvem essa síndrome durante internação na UTI.

\section{Material e Métodos}

Foram avaliados e acompanhados, de modo prospectivo, 263 pacientes adultos admitidos na UTI Central do HC-FMB durante o período de agosto de 2007 a abril de 2008, desde a internação até o dia da alta da UTI para leitos da enfermaria ou óbito. O acompanhamento foi apenas observacional, sem qualquer intervenção.

Diariamente, um dos responsáveis pela pesquisa visitou a UTI central e, para cada paciente internado, preencheu-se um protocolo com informações gerais sobre a identificação do paciente, as condições associadas à sua internação e o acompanhamento clínico e laboratorial (creatinina, ureia e potássio séricos) dos parâmetros de função renal, assim como o momento do diagnóstico de IRA realizado pela equipe de intensivistas.

Ao final do período de acompanhamento, os pacientes foram divididos em dois grupos, de acordo com a evolução da função renal apresentada durante a internação:

- Grupo 1: pacientes que apresentaram diagnóstico de IRA durante o período de internação na UTI;

- Grupo 2: pacientes que mantiveram função inalterada durante internação na UTI.

A IRA foi definida como condição clínica em que, a partir de um insulto renal, o paciente apresentou aumento de creatinina $(\mathrm{Cr}) \mathrm{de} 0,3 \mathrm{mg} / \mathrm{dL}$ se Cr basal $<1,5 \mathrm{mg} / \mathrm{dL}$ ou $>50 \%$ se Cr basal $>1,5 \mathrm{mg} / \mathrm{dL}$, ou se diurese inferior a $0,5 \mathrm{~mL} / \mathrm{kg} /$ hora por mais de 12 horas. ${ }^{25}$

Foram excluídos pacientes menores de 18 anos, portadores de Insuficiência Renal Crônica nos estágios IV e V, ${ }^{26}$ aqueles em tratamento dialítico crônico ou submetidos a transplante renal e pacientes com permanência inferior a 48 horas na UTI.
Na medida do possível, o observador não informou à equipe de médicos intensivistas sobre o desenvolvimento do trabalho, exceto para os responsáveis pela Unidade.

Este estudo recebeu parecer favorável do Comitê de Ética em Pesquisa da FMB-UNESP e foi aprovado em 3 de setembro de 2007.

\section{AnÁlise Estatística}

A partir do protocolo de estudo, os dados foram digitados em planilha eletrônica e verificados eventuais erros de digitação.

A análise dos dados foi feita com o auxílio do programa estatístico STATA 8.0 (STATACORP, 2004).

Inicialmente, realizou-se uma análise descritiva dos pacientes internados na UTI, avaliando-se a incidência de IRA durante o período de observação.

A seguir, foram estudados os pacientes divididos em dois grupos, de acordo com a presença ou não de IRA, fazendo-se análise descritiva e calculando-se as medidas de tendência central e dispersão para as variáveis contínuas e de frequência para as variáveis categóricas.

Em ambos os grupos, a significância estatística da associação entre as variáveis categóricas e a presença de IRA foi avaliada por meio do Teste do Qui-Quadrado, enquanto, entre as variáveis contínuas e o mesmo desfecho, a presença foi avaliada pelo Teste t. Em ambos, foi considerada significância estatística quando $\mathrm{p}<0,05$.

$\mathrm{Na}$ última etapa, realizaram-se comparações entre os grupos. Os dados contínuos com distribuição paramétrica foram analisados pelo Teste $t$ e os não paramétricos, pelo Teste de Mann-Whitney.

\section{Resultados}

Neste estudo, foram incluídos 297 pacientes, durante o período de agosto de 2007 a abril de 2008. Desses, 34 foram excluídos pelas seguintes razões: nove devido à permanência na UTI inferior a 48 horas, cinco por apresentarem IRC IV ou V, 11 por estarem em tratamento dialítico crônico ou submetidos a transplante renal. Portanto, na análise final, foram avaliados 263 pacientes, conforme mostra a Figura 1.

A incidência de IRA na UTI foi de $31,2 \%$. A seguir, os pacientes admitidos na UTI central durante o período de estudo foram divididos em dois grupos: G1 (com IRA) e G2 (sem IRA). Os grupos foram semelhantes em relação a sexo, conforme mostra a Figura 2.

A Figura 3 mostra que houve diferença estatisticamente significativa na distribuição dos pacientes com e sem IRA segundo a etiologia da admissão na UTI, com predomínio de sepse em G1 (31,7\% x 13,1\%, $\mathrm{p}<0,0001)$ e de pós- operatório em G2 (11\% x 43\%, 
$\mathrm{p}<0,0001)$ e também conforme a enfermaria de procedência (pronto-socorro: G1 $22 \times 14,5 \%, \mathrm{p}=0,02 \mathrm{e}$ centro cirúrgico: G1 42,7 x 62,6\%, p = 0,03).

Os grupos diferiram quanto à idade (G1 59,6 \pm 18,1 x G2 50,2 \pm 18,6 anos, p < 0,0001), APACHE II (G1 $21 \pm 11,1$ x G2 11 4,8, p = 0,002), oligúria (G1 67,7 x G2 4,5\%, p < 0,0001), presença de ventilação mecânica (G1 81,7 x G2 57,7\%, p = 0,0014) e necessidade de drogas vasoativas $(62,2 \times 32,6 \%$, $\mathrm{p}<0,0001)$, conforme mostra a Tabela 1 .

Figura 1. Pacientes eleitos para o estudo, admitidos na Unidade de Terapia Intensiva Central do HC-FMB entre agosto de 2007 e abril de 2008 e incluídos na análise final.

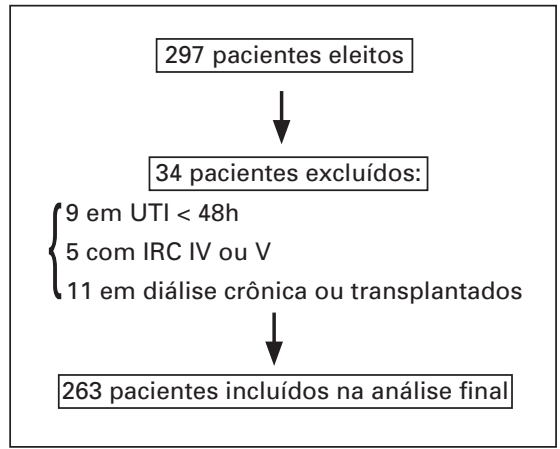

Figura 2. Distribuição dos pacientes com e sem Injúria Renal Aguda, de acordo com o sexo.

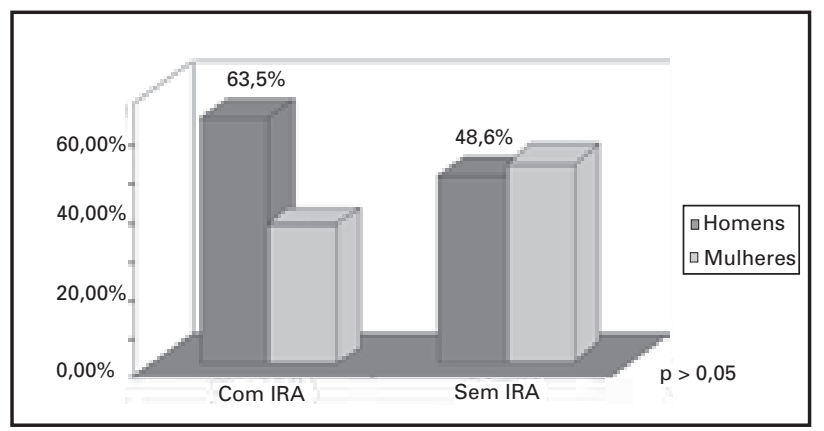

Figura 3. Distribuição dos pacientes com e sem Injúria Renal Aguda, conforme a etiologia da admissão na UTI central e a enfermaria de procedência.

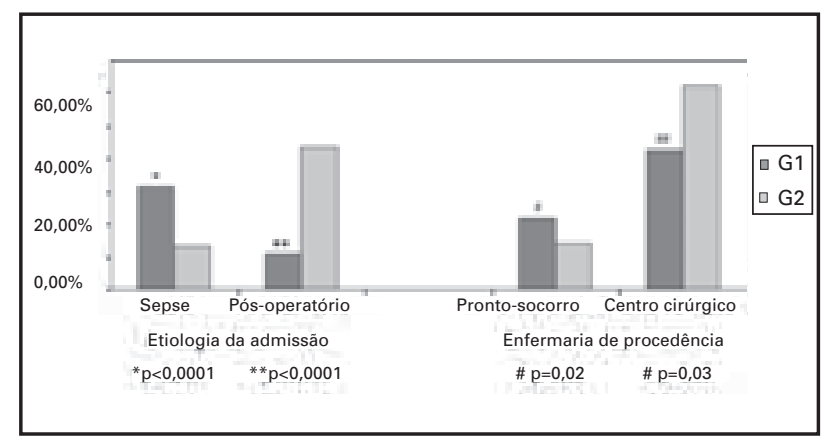

Figura 4. Distribuição dos pacientes com e sem Injúria Renal Aguda, conforme a presença de comorbidades.

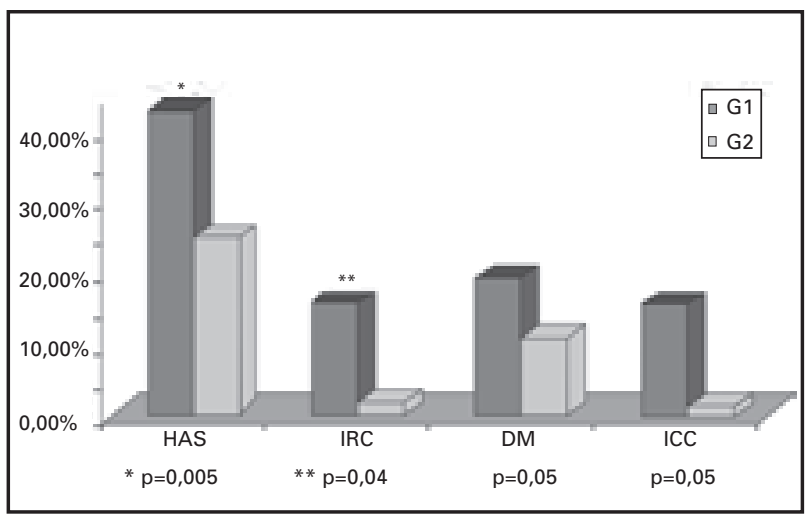

Figura 5. Distribuição dos pacientes com e sem Injúria Renal Aguda, conforme a mortalidade.

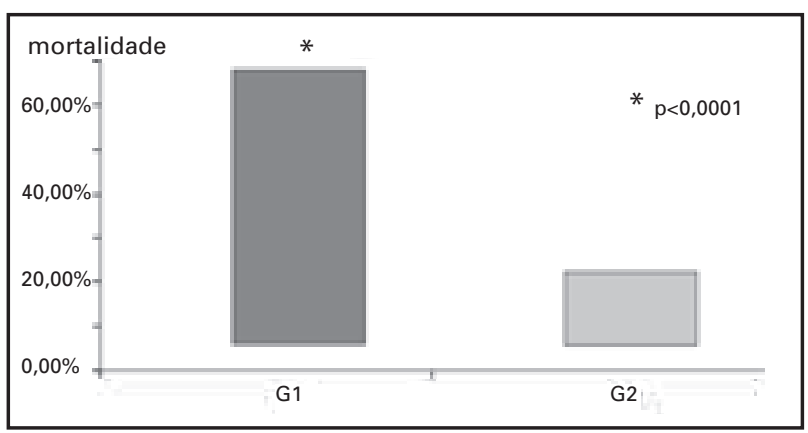

\begin{tabular}{|lccc|}
\hline Tabela 1 & $\begin{array}{l}\text { DISTRIBUIÇÃo DOS PACIENTES COM E SEM IRA DE ACORDO COM A IDADE, APACHE II, } \\
\text { PRESENÇA DE VENTILAÇĀO MECÂNICA E NECESSIDADE DE DROGAS VASOATIVAS }\end{array}$ & \\
\hline Grupo 1 & Grupo 2 & $\mathrm{p}$ \\
\hline Idade (anos) & $59,6 \pm 18,1$ & $50,2 \pm 18,6$ & 0,0001 \\
\hline APACHE II & $21 \pm 11,1$ & $11 \pm 4,8$ & 0,002 \\
\hline Oligúria (\%) & 67,5 & 4,5 & 0,0014 \\
\hline Ventilação Mecânica (\%) & 81,7 & 57,7 & $<0,0001$ \\
\hline Drogas Vasoativas (\%) & 62,2 & 32,6 & \\
\hline
\end{tabular}


Com relação às comorbidades, os grupos foram diferentes quanto à presença de hipertensão arterial e IRC $(42,6 \times 25,9 \%, p=0,005$ e $15,8 \times 2,1 \%$, $\mathrm{p}=0,04$, respectivamente) e semelhantes quanto à presença de diabetes e insuficiência cardíaca congestiva (G1: $19,5 \times 11 \%$, ns e 6 x 1,1\%, ns, respectivamente), conforme mostra a Figura 4.

A mortalidade dos pacientes internados na UTI foi superior no grupo que adquiriu IRA $(62,1 \times 16,5 \%$, $\mathrm{p}<0,0001)$, conforme mostra a Figura 5 .

\section{Discussão}

O presente trabalho teve como objetivos principais avaliar a incidência da IRA em pacientes admitidos na UTI central do HC-FMB e analisar comparativamente as características clínicas e a evolução dos pacientes que desenvolveram ou não IRA.

Segundo a literatura, a IRA é uma das mais importantes complicações observadas em pacientes que estão hospitalizados; sua incidência varia de acordo com as condições clínicas dos pacientes, sendo maior em UTIs (20 a 40\%). Em estudo recente, Hsu et al..$^{28}$ mostraram que a incidência da IRA com e sem necessidade de diálise vem aumentando progressivamente na última década. De 1996 a 2003, a incidência da IRA sem necessidade de diálise passou de 322,7 para $522,4 / 100.000$ pessoas/ano, enquanto a IRA com indicação dialítica de 19,5 para 29,5/100.000 pessoas/ ano). Como já descrito, a IRA, além de ser uma complicação comum em pacientes críticos, é um fator de risco independente de morte. ${ }^{1,3,8,12}$

Neste trabalho, de acordo com o descrito na literatura, a IRA foi frequente em UTI, ocorrendo em $31,2 \%$ dos pacientes admitidos.

De modo geral, estudos da literatura mostram que a presença de condições que determinam hipoperfusão e isquemia renal está diretamente relacionada ao desenvolvimento de IRA e que os pacientes que apresentam redução da reserva funcional renal são mais suscetíveis a desenvolver IRA mesmo com lesões renais de pequena monta. ${ }^{16,1}$ Dessa forma, consideramse fatores de risco para IRA idade avançada, nível prévio de creatinina, presença de diabetes mellitus, de hipertensão arterial e de insuficiência cardíaca congestiva, além do uso crônico de anti-inflamatórios não hormonais. ${ }^{16,17}$

Neste trabalho, o grupo com IRA apresentou resultados semelhantes ao descrito na literatura, ocorrendo em pacientes mais idosos e com mais comorbidades associadas, principalmente hipertensão arterial e IRC.
Muitos fatores contribuem para a manutenção da mortalidade elevada na IRA, com destaque para a falta de identificação de fatores de risco para o desenvolvimento desta patologia, assim como o desconhecimento de fatores associados à mortalidade. ${ }^{15}$

Liano et al. ${ }^{10}$ apontaram diferença significativa na mortalidade de pacientes com IRA internados em UTI (70 a $80 \%)$ e em unidades de cuidados intermediários (próximas de $50 \%$ ). Uma possível explicação para essa diferença é o perfil epidemiológico dos pacientes em UTI, que é mais grave, com predomínio de insuficiências de múltiplos órgãos, estado, em geral, associado à elevada mortalidade. Nessa condição clínica, raramente a IRA se apresenta como uma manifestação isolada, mas, sim, como uma complicação de um largo espectro de doenças. ${ }^{14,16}$ Neste trabalho, a mortalidade dos pacientes que adquiriram IRA foi de $62,1 \%$, estatisticamente superior à observada no grupo sem IRA, que foi de $16,5 \%(\mathrm{p}<0,0001)$. Isso pode ser justificado pela presença de parâmetros clínicos e índices prognósticos de maior gravidade encontrados nos pacientes com IRA, ou seja, eram mais idosos, mais oligúricos, com APACHE II mais elevado, além da maior necessidade de drogas vasoativas e de ventilação mecânica.

Em resumo, neste estudo, a IRA foi frequente em UTI, ocorrendo em pacientes mais graves, ou seja, com parâmetros clínicos e índices prognósticos de maior gravidade, o que justifica a maior mortalidade observada neste grupo.

Porém, mais estudos prospectivos são necessários para identificar os fatores de risco associados ao desenvolvimento e ao prognóstico desfavorável da IRA para que medidas preventivas e de diagnóstico precoce possam ser realizadas. ${ }^{16,17}$

\section{ReferênCIAS}

1. Brady HR, Brenner BM, Clarkson MR, Lieberthal W. The Kidney. In: Brenner BM, editor. Acute renal failure. Philadelphia: Saunders 2000; 1201-62.

2. Bellomo R, Ronco C, Kellum JA et al. Acute renal failure definition, outcome measures, animal models, fluid therapy and information technology needs: the Second International Consensus Conference of the Acute Dialysis Quality Initiative (ADQI) Group. Crit Care 2004; 8:204-11.

3. Brivet FG, Kleinknecht DJ, Loirat P, Landais PJ. Acute renal failure in intensive care units: causes, outcome and prognostic factors of hospital mortality: a prospective, multicenter study. Crit Care Med 1996; 24:192-8.

4. Lameire N, Hoste E. Reflections on the Definition, Classification, and Diagnostic Evaluation of Acute Renal Failure. Curr Opin Critl Care 2004; 10:468-75. 
5. Ricci Z, Ronco C, D’amico G et al. Practice Patterns in the Management of Acute Renal Failure in the Critically Ill Patient: An International Survey. Nephrol Dial Transplant 2006; 21:690-6.

6. Han WK, Bonventre JV. Biologic Markers for the Early Detection of Acute Kidney Injury. Curr Opin Crit Care 2004; 10:476-82.

7. Mehta RL, Kellum JA, Shah SV et al. the Acute Kidney Injury Network: report of an initiative to improve outcomes in acute kidney injury. Crit Care 2007; 11:R31.

8. Mehta RL, Chertow GM. Acute Renal Failure Definitions and Classification: Time for Change? J Am Soc Nephrol 2003; 14:2178-87.

9. Clermont G, Acker DC, Sirio CA, Pinsky MR, Johnson JP. Renal failure in ICU: comparison of the impact of acute renal failure and end-stage renal disease on ICU outcomes. Kidney Int 2002; 62:986-96.

10. Liano F, Junco E, Pascual J et al. The spectrum of acute renal failure in the intensive care unit compared with that seen in others settings. The Madrid Acute Renal Failure Study Group. Kidney Int Suppl 1998; 66:S1624.

11. Lameire N, Van Biesen W, Vanholder R. Acute Renal Failure. Lancet 2005; 5:417-30.

12. Uchino S, Kellum JA, Bellomo $\mathrm{R}$ et al. Beginning and Ending Supportive Therapy for the Kidney (BEST Kidney) Investigators. Acute Renal Failure in Critically Ill Patients: A Multinational, Multicenter Study. JAMA 2005; 294:813-8.

13. Mehta RL, Pascual MT, Soroko S et al. Spectrum of Acute Renal Failure in the Intensive Care Unit: The PICARD Experience. Kidney Int 2004; 66:1613-21.

14. Barretti P, Soares VA. Acute Renal failure: clinical outcome and causes of death. Ren Fail 1997;19:253-7.

15. Santos WJ, Zanetta DM, Pires AC, Lobo SM, Lima EQ, Burdmann EA. Patients with ischaemic, mixed and nephrotoxic acute tubular necrosis in the intensive care unit - a homogeneous population? Crit Care 2006; 10:R68.

16. Balbi AL, Ponce D, Silva VS, Silva RPS, Torres AS, Barsante RC. Insuficiência Renal Aguda: análise de 500 pacientes atendidos em Hospital Universitário. J Bras Nefrol 2003; S1:50.
17. Liano F, Gallego A, Pascual J et al. Prognosis of acute tubular necrosis: an extended prospectively contrasted study. Nephron 1993; 63:21-3.

18. Himmelfarb J, Ikizler TA. Acute kidney injury: changing lexicography, definitions, and epidemiology. Kid Int 2007; 71:971-6.

19. Liãno F, Junco E, Pascual J et al. The spectrum of acute renal failure in the intensive care unit compared with that seen in other settings. Kid Int 1998, 53:16-24.

20. Kellum JA, Ronco C, Mehta R, Bellomo R. Consensus Development in Acute Renal Failure: the Acute Dialysis Quality Initiative. Curr Opin Crit Care 2005; 11:52732.

21. Maccariello E, Soares M, Valente C et al. RIFLE classification in patients with acute kidney injury in need of renal replacement therapy. Intensive Care Med 2007; 33:597-605.

22. Balbi AL, Ponce D, Silva VS, Silva RPS, Torres AS, Barsante RC. Insuficiência Renal Aguda: análise de 500 pacientes atendidos em Hospital Universitário. J Bras Nefrol 2003; S1:50.

23. Abosaif NY, Tolba YA, Heap M, Russell J, El Nahas AM. The outcome of acute renal failure in the intensive care unit according to RIFLE: model application, sensitivity, and predictability. Am J Kidney Dis 2005; 46:1038-48

24. Sesso R, Roque A, Vicioso B et al. Prognosis of ARF in Hospitalized Elderly Patients. Am J Kid Dis 2004; 44:410-9.

25. Mehta RL, Kellum JA, Shah SV et al. The Acute Kidney Injury Network: report of an initiative to improve outcomes in acute kidney injury. Crit Care 2007; 11:R31.

26. Romão JEJ. Doença Renal Crônica: Definição, Epidemiologia e Classificação. J Bras Nefrol 2004; 26(Supl 1):1-3.

27. Himmelfarb J, Ikizler TA. Acute kidney injury: changing lexicography, definitions, and epidemiology. Kid Int 2007; 71:971-6.

28. Hsu CY, McCulloch CE, Fan D et al. Communitybased incidence of acute renal failure. Kid Int 2007; 72:208-12. 